\title{
Histerektomi Olgularında Adenomyosis Prevelansı ve İlişkili Faktörler
}

The Prevelance of Adenomyosis at Hysterectomy and Associated Factors

\section{Mehmet Baki Şentürk ${ }^{1}$, Mehmet Şükrü Budak ${ }^{2}$, Yusuf Çakmak ${ }^{3}$ Ömer Birol Durukan ${ }^{4}$, Mesut Polat ${ }^{4}$}

\author{
1. Bakırköy Dr. Sadi Konuk Eğitim ve Araştırma Hastanesi, Kadın Hastalıkları ve Doğum Kliniği İstanbul, Türkiye. \\ 2. Diyarbakır Doğumevi, Kadın Hastalıkları ve Doğum Kliniği, İstanbul, Türkiye. \\ 3. Batman Devlet Hastanesi, Batman, Türkiye. \\ 4. Zeynep Kamil Kadın ve Çocuk Hast. Eğt. ve Arş. Hast., Kadın Hastalıları ve Doğum Kliniği, İstanbul, Türkiye.
}

\section{$\ddot{O} Z E T$}

Amaç: Adenomyosis, endometrial gland ve stromanın endometriumla ilişkisiz bir șekilde myometrium içine gömülmesidir. Tanısı histolojik incelemeye dayandiğ $\mathrm{için,} \mathrm{bu} \mathrm{durum} \mathrm{en} \mathrm{iyi} \mathrm{histerektomi} \mathrm{materyal-}$ lerinin incelenmesi ile belirlenmektedir.

Gereç ve Yöntemler: Bu çalışmada histerektomi olmuş 109 hastanın sonuçları retrospektif olarak incelendi. Yaş, parite, menapozal durum ve histerektomi endikasyonlar adenomyosis olan ve olmayan grup arasında karşılaştırıldi. P değerinin 0,05 altında olmast istatistiksel olarak anlamlı kabul edildi.

Bulgular: Adenomyosis olan olgularin ortalama yaşı 55 idi. Parite ve anormal uterin kanama adenomyosis olan olgularda daha yüksek idi $(p<0,05)$.

Sonuç: Bu çalışmada histerektomi materyallerindeki adenomyosis oranı \% 29,4 olarak görüldü. Anormal uterin kanaması olan ve parite sayıs fazla olan olgularda adenomyosis oranı daha sık görülmüşı̈̈r.

Anahtar Kelimeler: adenomyosis; histerektomi

\section{ABSTRACT}

Objective: Adenomyosis is a entity characterized by the presence of endometrial glands and stroma embedded within the myometrium without apparent contact with the endo-myometrial junction. As the diagnosis of adenomyosis is based on histological examination, the condition is best described in women at the time of hysterectomy.

Material and Method: We evaluated results of 109 hysterectomized patients retrospectively. Age, parity, menopausal status and indication of hysterectomy were compared between adenomyosis positive and negative patients according to histological findings. $P$ value under 0.05 was considered statistically significant.

Results: The mean of age patients with adenomyosis were 55. Parity and abnormal uterine bleeding were associated factors with adenomyosis $(p<0.05)$.

Conclusion: Our study demonstrated that the prevalence of adenomyosis at hysterectomy was 29.4\%. Adenomyosis is more frequent among women reporting abnormal uterine bleeding and with high parity.

Keywords: adenomyosis; hysterectomy

\section{İletişim Bilgileri:}

Sorumlu Yazar: Mehmet Baki ŞENTÜRK

Yazışma Adresi: Bakırköy Dr. Sadi Konuk Eğitim ve Arş. Hastanesi, Tevfik Sağlam Cad., No: 11, Zuhuratbaba Bakırköy 34147, İstanbul

E-mail: dr.baki77@gmail.com

Makalenin Geliş Tarihi: 04.02.2015

Makalenin Kabul Tarihi: 13.08.2015

DOI: http://dx.doi.org/10.16948/zktb.11649 


\section{GİRIŞ}

Adenomyosis endometrial gland ve stromanın myometrium içerisinde olsuşturduğu lezyondur [1]. S1k semptomları kronik pelvik ağr1, menoraji ve dismenoredir [2]. Histerektomi materyallerinin incelenmesinde prevelans1 $\% 8.8$ ile 61.5 arasında değişmektedir [3]. Adenomyosisin preoperatif tanısını koymak güçtür, histerektomi matermallerinin incelenmesinde rastlantısal olarak tanı konduğu ve tipik semptomlara neden olmadığı öne sürülmektedir [4]. Bununla beraber magnetic resonans ve transvaginal ultrasonografi uterustaki lezyonların karakterini ortaya koymada yararlı bulunmuştur $[5,6]$. Adenomyosisin nedeni tam olarak bilinmemektedir fakat, hormonal (östrojen gibi), genetik, immünolojik ve büyüme faktörleri gibi değișik faktörler çeșitli çalıșmalar sonucunda neden olarak önerilmiştir [7].

Bu çalışmada değişik endikasyonlarla yapilan 109 histerektomi materyalindeki adenomyosis oranı retrospektif olarak incelenerek adenomyosis ile ilişkili faktörler araştırıldı.

\section{MATERYAL ve METOD}

Diyarbakır Kadın Doğum ve Çocuk Hastaliklar1 Hastanesinde 01.01.2012 ile 31.12.2013 tarihleri arasında değişik endikasyonlarla yapılan 109 histerektomi materyalinin patolojik sonuçları retrospektif olarak incelendi. Hastaların yaş, gravida, menapoz durumu kaydedildi. Histerektomi materyallerindeki patoloji sonuçları ile beraber adenomyosis oranı ile beraber ilişkili faktörler araştırıldı. İstatistiksel analizler için NCSS (Number Cruncher Statistical System) 2007\&PASS (Power Analysis and Sample Size) 2008 Statistical Software (Utah, USA) programı kullanıldı. Adenomyosisisin yaş, perite, menapoz durumu, anormal uterin kanama, prolapsus, uterin atoni, postmenapozal kanama ve myom ile ilişkisini araştırmak için Yates' Continuity Correction test (Yates düzeltmeli Ki-kare), yaşa göre Student t test, pariteye göre Mann Whitney U test, Fisher's Exact test kullanıld1. Anlamlılık $p<0.05$ düzeyinde değerlendirildi.

\section{SONUÇ}

Diyarbakır Kadın Doğum ve Çocuk Hastaliklar1 Hastanesinde 01.01.2012 ile 31.12.2013 yılları arasında bening endikasyonlarla histerektomi yapılmıs 109 olgunun patolojik sonuçları incelendi. Adenomyosis 32 hastada görüldü (\%29.4). Hastaların demografik özellikleri, menopoz durumu, preoperatif tanı ve histerektomi teknikleri Tablo 1'de görülmektedir.
Tablo 1. Olguların demografik özellikleri, preoperatif tanı ve yapıımış histerektomi yöntemleri.

\begin{tabular}{|c|c|c|c|}
\hline & & Min-Mak & Ort $\pm S D$ \\
\hline Yaş (yıl) & & $15-84$ & $53,53 \pm 14,01$ \\
\hline Parite & & $0-15$ & $7,64 \pm 3,00$ \\
\hline & & $\mathrm{n}$ & $\%$ \\
\hline Menop & & & \\
\hline & Var & 57 & 52,3 \\
\hline & Yok & 52 & 47,7 \\
\hline Preop T & & & \\
\hline & Genital prolapsus & 52 & 47,7 \\
\hline & AUK & 27 & 24,8 \\
\hline & Atoni & 11 & 10,1 \\
\hline & PMK & 10 & 9,2 \\
\hline & PMK + Genital prolapsus & 3 & 2,8 \\
\hline & AUK + Myom & 2 & 1,8 \\
\hline & AUK + Genital prolapsus & 1 & 0,9 \\
\hline & AUK + Kr pelvik ağrı & 1 & 0,9 \\
\hline & Postparum geç hemoraji & 1 & 0,9 \\
\hline & Rüptür & 1 & 0,9 \\
\hline
\end{tabular}

\begin{tabular}{|l|l|l|l|}
\hline Operasyon Tipi & & \\
\hline & VAH & 55 & 50,5 \\
\hline & TAH & 21 & 19,3 \\
\hline & LTH & 18 & 16,5 \\
\hline & Subtotal histerektomi & 14 & 12,8 \\
\hline & LVAH & 1 & 0,9 \\
\hline
\end{tabular}

Patoloji sonuçlarına göre adenomyosis oran1 atrofik endometrium' dan sonra ikinci siklıkta $\% 29,4(n=32)$ olarak görülmektedir (Tablo 2$)$. 
Tablo 2. Patoloji sonuçları.

\begin{tabular}{|c|c|c|}
\hline & $\mathbf{n}$ & $\%$ \\
\hline Atrofik endometrium & 29 & 26,6 \\
\hline Adenomyozis & 20 & 18,3 \\
\hline Gestasyonel hemorajik endometrium & 12 & 11,0 \\
\hline Sekretuar endometrium & 9 & 8,3 \\
\hline Proliferatif endometrium & 7 & 6,4 \\
\hline Adenomyozis+atrofik endometrium & 3 & 2,8 \\
\hline Atrofik endometrium+myom & 3 & 2,8 \\
\hline Düzensiz prolıferatıf endometrium & 3 & 2,8 \\
\hline Adenomyozis+basit atipisiz hiperplazi & 2 & 1,8 \\
\hline Adenomyozis+endometrial polip & 2 & 1,8 \\
\hline Myom+sekretuar endometrium & 2 & 1,8 \\
\hline Myoma uteri & 2 & 1,8 \\
\hline Proliferatif endometrium+myom & 2 & 1,8 \\
\hline Adenomyosis+atrofik endometrium+polip & 1 & 0,9 \\
\hline Adenomyozis+adenomyomatoz polip & 1 & 0,9 \\
\hline Adenomyozis+myom & 1 & 0,9 \\
\hline $\begin{array}{l}\text { Adenomyozis+myom+düzensiz proliferatif } \\
\text { endometrium }\end{array}$ & 1 & 0,9 \\
\hline Atrofik uterus & 1 & 0,9 \\
\hline Basit atipisiz hiperplazi & 1 & 0,9 \\
\hline Endometrit & 3 & 2.8 \\
\hline Myom+endometrial polip & 1 & 0,9 \\
\hline Myom+proliferatif endometrium & 1 & 0,9 \\
\hline Proliferatif endometriyum+adenomyosis & 1 & 0,9 \\
\hline Sekretuar endometrium+leiomyomatozis & 1 & 0,9 \\
\hline
\end{tabular}

Adenomyosis görülen olguların pariteleri daha yüksek ( $\mathrm{P}=0.0 .38)$, anormal uterin kanama kliniği daha fazla $(\mathrm{p}=0.026)$ iken atoni tanisı ile yapılan histerektomilerde adenomyosis görülme oranı daha az bulunmuştur $(\mathrm{p}=0.032)$. Diğer parametreler ile adenomyosis arasında ilişki saptanmamıştır ( $>0.05$ ) (Tablo 3).
Tablo 3. Adenomyosisin klinik, yaş, parite ve menapoz ile ilişkisi.

\begin{tabular}{|c|c|c|c|c|}
\hline & & \multicolumn{2}{|c|}{ Patoloji Sonucu } & \\
\hline & & $\begin{array}{l}\text { Adenomyo- } \\
\text { zis }(-)(n=77)\end{array}$ & $\begin{array}{l}\text { Adenomyo- } \\
\text { zis }(+)(n=32)\end{array}$ & \\
\hline & & $\mathrm{n}(\%)$ & $\mathrm{n}(\%)$ & $p$ \\
\hline \multicolumn{2}{|c|}{ Genital prolapsus } & $40(51,9)$ & $12(37,5)$ & ${ }^{\mathrm{a}} 0,244$ \\
\hline \multicolumn{2}{|l|}{ AUK } & $14(18,2)$ & $13(40,6)$ & ${ }^{\mathrm{a}} 0,026^{*}$ \\
\hline \multicolumn{2}{|l|}{ Atoni } & $11(14,3)$ & $0(0)$ & ${ }^{\mathrm{b}} 0,032 *$ \\
\hline \multicolumn{2}{|l|}{ PMK } & $6(7,8)$ & $4(12,5)$ & ${ }^{\mathrm{b}} 0,475$ \\
\hline \multicolumn{2}{|c|}{$\begin{array}{l}\text { Genital prolapsusu } \\
+ \text { PMK }\end{array}$} & $1(1,3)$ & $2(6,3)$ & ${ }^{\mathrm{b}} 0,206$ \\
\hline \multicolumn{2}{|l|}{ AUK + myom } & $1(1,3)$ & $1(3,1)$ & ${ }^{\mathrm{b}} 0,503$ \\
\hline \multicolumn{2}{|c|}{$\begin{array}{l}\text { AUK + genital } \\
\text { prolapsus }\end{array}$} & $1(1,3)$ & $0(0)$ & b1,000 \\
\hline \multicolumn{2}{|l|}{$\begin{array}{l}\text { AUK + Kr } \\
\text { pelvik ağrı }\end{array}$} & $1(1,3)$ & $0(0)$ & ${ }^{b} 1,000$ \\
\hline \multicolumn{2}{|l|}{$\begin{array}{l}\text { Postparum } \\
\text { geç hemoraji }\end{array}$} & $1(1,3)$ & $0(0)$ & ${ }^{b} 1,000$ \\
\hline \multicolumn{2}{|l|}{ Rüptür } & $1(1,3)$ & $0(0)$ & ${ }^{b} 1,000$ \\
\hline \multirow{2}{*}{ Menapoz } & $\operatorname{Var}(n=57)$ & $40(51,9)$ & $17(53,1)$ & \multirow{2}{*}{${ }^{\mathrm{a}} 1,000$} \\
\hline & Yok $(n=52)$ & $37(48,1)$ & $15(46,9)$ & \\
\hline Yaş (yıl) & \multirow{2}{*}{$\begin{array}{c}7.27 \pm \\
2.96(7)\end{array}$} & $\begin{array}{c}52,79 \pm \\
15,44\end{array}$ & $\begin{array}{c}55.31 \pm \\
9,70\end{array}$ & ${ }^{\circ} 0,308$ \\
\hline $\begin{array}{c}\text { Parite; } \\
\text { (medyan) }\end{array}$ & & $\begin{array}{c}7,27 \pm \\
2,96(7)\end{array}$ & $\begin{array}{c}8,53 \pm \\
2,96(8,5)\end{array}$ & ${ }^{d} 0,038^{*}$ \\
\hline
\end{tabular}

\section{TARTIŞMA}

$\mathrm{Bu}$ çalışma sonucunda bening nedenlerle yapilan histerektomi materyallerinin patoloji sonuçlarına göre adenomyosis oranı \%29.4 olarak görülmüsstür ve bu oran literatürde bildirilen çalışmlarla örtüşmektedir [8,9]. Bununla beraber adenomyosis en kesin tanisı uterusun tamamının histolojik incelenmesine dayanmaktadır. Histerektomi endikasyonu toplumlar arasında büyük değişkenlikler gösterdiğinden adenomyosis prevelansı dünya çapında büyük değişkenliler göstermektedir [10]. Tüm uterin dokunun ayrint1lı incelenmesi bile prevelansını etkilemektedir çünkü endomyometrial bileşkeden adenomyosis odağına kadar olan dokunun bölümlenerek incelenmesine dayanan sonuçlarda prevelans \%10 ile \%18.2 arasında değişmektedir [9]. Çalı̧̧ma sonucunda parite sayısının 
fazla olması ile adenomyosis arasında ilișki görülmüştür $(p<0.05)$. Bu sonuç literatürde bildirilen çalışmlaarla örtüşmektedir [11-13]. Endometrial dokunun mekanik hasarına neden olabilecek sebepler sonucu adenomyosis riski artmaktadır. Duğum sayısının artması, abortus indüksiyonu, sezeryan sayısının artması riski artırmaktadır $[12,14,15,13,16,17,18]$. Endometrial dokunun gerek doğum, gerek abortus ve gerekse sezeryan sonrasında bütünlüğünün bozulması ve endometrial hücrelerin burdan mekanik transportu endometriosise neden olabiilmektedir [11]. Bununla beraber abortus ya da sezeryan ile riskin artmadığını söyleyen çalışmalar da vardır $[11,19,14,20]$. Bu çalışmada adenomyosis ile ilişkili diğer bir faktör anormal uterin kanamadır. Fakat bazı çalışmalarda bu ilișkis saptanmamıștır [8, 9, 11]. Myometrial dokunun invazyonunu artması uterusun büyümesine ve kasilmasının bozulmasına neden olarak özellikle de mens döneminde aşırı kanmaya neden olabilir. Ayrıca östrojen ile adenomyosis arasında pozitif korelasyon vardır ve yüksek konsantrasyonda östrojen adenomyosisli hastalarda menstrüel kanamanın artışına neden olabilir $[12,21]$. Bu çalışmada adeomyosisli hastaların yas ortalaması 55 olarak görülmektedir. $\mathrm{Bu}$ literatürde daha önce bildiirilen çalıșmalarla örtüşmektedir [9, 11, 19, 13]. Bununla beraber Taran ve arkadaşları kendi çalıșmalarında adenomyosisli hastalardaki histerektomi için yaş ortalamalarını daha genç (44 yaş) olarak bildirmişlerdir [8]. Bu durum histerektomi endikasyonlarının dağılımı ile ilişkili olabilir. Çünkü bu çalışmada endikasyonların büyük çoğunluğunu (\%47.7) genital prolapsus hastaları içermekte ve bu hasta grubu görece yaşlı hastalardan oluşmaktadır. Ayrıca çalışmamamızda adenomyosis ile yaş arasında ilişki görülmemiştir $(p>0.05)$. Postmenapozal 17 hastada adenomyosis görülmüştür fakat menopoz durumuna göre adenomyosis sıklığında değişiklik saptanmamıştır $(\mathrm{p}>0.05)$. Adenomyosis ile östrojen arasında pozitif korelasyon olduğundan postmenapozal dönemde azalan östrojenle beraber adenomyotik odaklarda atrofi gelişir ve insidansı postmenapozal dönemde azalır $[12,21]$. Calışmanın retrospektif olması ve gorece az olgu içermesi limitasyonlarıdır. Çalışma sonucu göstermiştir ki parite ve anormal uterin kanama adenomyosis ile ilişkili bulunmuştur ve insidansı ileri yaşlarda artmaktadır.

\section{KAYNAKLAR}

1. Ferenczy A. Pathophysiology of adenomyosis. Hum Reprod Update .1998;4(4):312-22.

2. Azziz R. Adenomyosis: current perspectives. Obstet Gynecol Clin North Am 1989; 16(1):221-35.
3. Hunter WC, Smith LL, Reiner WC. Uterine adenomyosis; incidence, symptoms, and pathology in 1,856 hysterectomies. Am J Obstet Gynecol 1947;53(4):663-8.

4. Weiss G, Maseelall P, Schott LL, Brockwell SE, Schocken M, Johnston JM. Adenomyosis a variant, not a disease? Evidence from hysterectomized menopausal women in the Study of Women's Health Across the Nation (SWAN). Fertil Steril 2009;91:201-6.

5. Mounsey AL, Wilgus A, Slawson DC. Diagnosis and management of endometriosis. Am Fam Physician 2006; 74,594-600.

6. Tahlan A, Nanda A, Mohan H. Uterine adenomyoma: a clinicopathologic review of 26 cases and a review of the literature. Int. J.Gynecol. Pathol. 2006;25, 361-365.

7. Saremi A, Bahrami H, Salehian P, HakakN, Pooladi A.Treatment of adenomyomectomy in women with severe uterine adenomyosis using a novel technique. Reprod Biomed Online 2014;28(6):75360.

8. Taran FA, Wallwiener M, Kabashi D, Rothmund R, Rall K, Kraemer $B$, et al. Clinical characteristics indicating adenomyosis at the time of hysterectomy: a retrospective study in 291 patients. Arch Gynecol Obstet 2012;285(6):1571-6

9. Bergholt T, Eriksen L, Berendt N, Jacobsen M, Hertz JB Prevalence and risk factors of adenomyosis at hysterectomy. Hum Reprod 2001;16(11):2418-21.

10. Haas S, Acker D, Donahue C, Katz ME.Variation in hysterectomy rates across small geographic areas of Massachusetts. Am J Obstet Gynecol 1993;169(1):150-4.

11. Parazzini F, Mais V, Cipriani S, Busacca M, Venturini P; GISE. Determinants of adenomyosis in women who underwent hysterectomy for benign gynecological conditions: results from a prospective multicentric study in Italy. Eur J Obstet Gynecol Reprod Biol 2009;143(2):103-6

12. Parazzini FVP, Panazza S, Chatenoud L, Oldani S, Crosignani PG. Risk factors for adenomyosis. Hum Reprod 1997;12:1275-9.

13. Vercellini P, Vigano P, Somigliana E, Daguati R, Abbiati A, Fedele L. Adenomyosis: epidemiological factors. Best Pract Res Clin Obstet Gynaecol 2006;20:465-77.

14. Levgur M, Abadi MA, Tucker A. Adenomyosis: symptoms, histology, and pregnancy terminations. Obstet Gynecol 2000;95:688-91.

15. Olive DL, Franklin RR, Gratkins $L V$. The association between endometriosis and spontaneous abortion. A retrospective clinical study. J Reprod Med 1982;27:333-8.

16. Vercellini P, Parazzini F, Oldani S, et al. Adenomyosis at hysterectomy: a study on frequency distribution and patient characteristics. Hum Reprod 1995; 10:1160-2.

17. Azziz R. Adenomyosis in pregnancy. A review. J Reprod Med 1986;31:224-7.

18. Merrill JA, Creasman WT. Disorders of the uterine corpus. In: Scott JR, Di Sala PJ, Hammond CB, et al., editors. Danforth's obstetrics and gynecology. 6th ed., Philadelphia: Lippincott; 1990. p. 1023.

19. Taran FA, Weaver AL, Coddington CC, Stewart EA Understanding adenomyosis: a case control study. Fertil Steril 2010;94(4):1223-8.

20. Panganamamula UR, Harmanli OH, Isik-Akbay EF, Grotegut CA, Dandolu V, Gaughan JP. Is prior uterine surgery a risk factor for adenomyosis? Obstet Gynecol 2004;104:1034-8.

21. Takahashi K, Nagata H, Kitao M. Clinical usefulness of determination of estradiol level in the menstrual blood for patients with endometriosis. Nihon Sanka Fujinka Gakkai Zasshi 1989;41(11):1849-50. 